\title{
The Number of Lymph Nodes and Relationship with Presence of Thyroiditis and Thymic Tissue in the Central Neck Dissection Materials for Thyroid Papillary Carcinoma: Pathologic Analysis
}

\author{
Banu Bilezikçi1* ${ }^{*}$, Seyfettin Ilgan², Serdar Özbaş³, Savaş Koçak ${ }^{3}$ \\ ${ }^{1}$ Departments of Pathology, Guven Hospital, Ankara, Turkey \\ ${ }^{2}$ Departments of Nuclear Medicine, Guven Hospital, Ankara, Turkey \\ ${ }^{3}$ Departments of Endocrine Surgery, Guven Hospital, Ankara, Turkey \\ Email: ‘banubil@yahoo.com
}

How to cite this paper: Bilezikçi, B., Ilgan, S., Özbaş, S. and Koçak, S. (2016) The Number of Lymph Nodes and Relationship with Presence of Thyroiditis and Thymic Tissue in the Central Neck Dissection Materials for Thyroid Papillary Carcinoma: Pathologic Analysis. International Journal of Clinical Medicine, 7, 566-576.

http://dx.doi.org/10.4236/ijcm.2016.79062

Received: July 10, 2016

Accepted: August 16, 2016

Published: August 19, 2016

Copyright $\odot 2016$ by authors and Scientific Research Publishing Inc. This work is licensed under the Creative Commons Attribution International License (CC BY 4.0).

http://creativecommons.org/licenses/by/4.0/ (c) (i) Open Access

\begin{abstract}
Background: Central lymph node dissection (CLND) for papillary thyroid carcinoma (PTC) allows correct pathologic staging of lymph nodes and planning of postoperative management. The purpose of this study was to determine the number of the lymph nodes in the CLND and the relationship to presence of chronic lymphocytic thyroiditis (CLT) and thymic tissue (TT). Methods: Total thyroidectomy and CLND materials from 153 patients with PTC were included in this study. Two histopathologic features (presence of CLT and TT) were evaluated for their value in adequacy of CLND. Results: Histopathologic examination revealed CLT and TT in CLND materials in $70(46 \%)$ and $63(41 \%)$ patients, respectively. Total number of lymph nodes in CLND materials was significantly higher in CLT $(+)$ and TT $(+)$ groups $(\mathrm{p}<0.001)$, independently. LN metastases in central compartment were found in $40 \%$ of patients $(\mathrm{n}=61)$. Of these, 30 patients had underlying CLT. The metastatic LN ratio (metastatic LN/total LN) was significantly lower in CLT (+) group, comparing to CLT (-) group $(16.8 \% \pm 12.2 \%$ vs $46.4 \% \pm 27.5 \%$; $<<0.001)$. Conclusions: Our study demonstrates that presence of CLT in thyroid gland has been associated with higher number of central lymph nodes mainly due to increased number of benign hyperplastic lymph nodes. It may be possible to conclude that upper limit of lymph nodes for satisfactory CLND would be higher to correctly evaluate central lymph node status in existing staging systems if specimens have CLT. Results of this study also show that the presence of TT in surgical materials may represent the adequacy of CLND.
\end{abstract}

\section{Keywords}

Thyroid Papillary Carcinoma, Central Lymph Node Dissection, Thyroiditis, Thymus 


\section{Introduction}

Regional lymph node (LN) metastasis is a common finding at first diagnosis in papillary thyroid carcinoma (PTC) occurring in $20 \%$ to $90 \%$ of cases depending on the surgical and histological techniques [1]. The LNs in central compartment of the neck (level VI) are involved most frequently [2]. While compartment oriented LN dissection is the favored surgical approach for preoperatively proven lateral cervical and central LN metastases, controversy exist for clinically silent nodal disease. It has generally been accepted that LN metastases at presentation increase the risk of locoregional tumor recurrence, but do not adversely affect survival in patients with PTC. However, the findings of a large population-based study show significantly higher mortality rates for PTC involving lymph nodes [3]. Similarly, recent studies shows that central LN metastasis is also an important prognostic factor in patients with PTC and routine central lymph node dissection (CLND) reduces recurrent/persistent disease and postoperative thyroglobulin levels [2] [4].

Since, preoperative physical examination and ultrasonography, and intraoperative palpation do not seem sensitive enough; routine CLND remains the only reliable method to identify the status of central LNs [5]. Despite potential increase in perioperative complication rates, mainly permanent hypoparathyroidism and recurrent laryngeal nerve dysfunction, routine CLND has also potential to lessen the need for adjuvant radioiodine therapy by providing possible surgical cure and accurate staging.

The extent of CLND and macroscopic examination of dissection materials should be adequate for an accurate nodal staging of central compartment. Additionally, the total number of nodes found is critical since it reflects both the extent of the node dissection and the care with which the pathologic examination was carried out [6]. Several clinicopathologic parameters including surgeon experience, surgical techniques and length of fresh CLND specimen have been already studied as factors for adequacy of CLND materials [7]-[10]. However, impacts of underlying chronic lymphocytic thyroiditis (CLT) as well as existence of thymic tissue (TT) in CLND material on adequacy of central dissection are unknown. Current study aims to define effects of CLT and existence of TT on adequacy of central dissection under standardized surgical and pathological approach.

\section{Material and Methods}

This study was approved by the Institutional Ethical and Scientific Committee of Guven Hospital. Data were retrieved from retrospectively maintained surgical and pathology databases.

\subsection{Selection of Patients}

Patients included in the study had proven PTC in all sizes. The diagnosis of PTC was made by fine-needle aspiration biopsy and reconfirmed by the surgical pathology according to characteristics nuclear features and diagnostic structural patterns in all patients. Preoperative LN status in lateral cervical chain was determined by routine neck 
ultrasonography and all recorded lymph nodes with suspicious sonographic features were further investigated accordingly. Standard approach to PTC without documented lateral cervical LN metastasis in our hospital is total thyroidectomy and CLND regardless of preoperative clinical and ultrasonographic findings. Lateral cervical LN dissection was added to standard surgical procedure for patients with proven LN metastasis in lateral compartment (23 patients), though results of lateral LN dissection were not taken into account.

From January 2012 to September 2014, a total of 153 consecutive patients, diagnosed as PTC and underwent total thyroidectomy plus CLND, were included in this study. Patients were excluded from the study if they had thyroid/parathyroid surgeries previously. All operations were performed by the same surgical team headed by one of our senior endocrine surgeons (SK, SO).

\subsection{Surgery}

Total thyroidectomy was performed by extracapsular dissection and the operation also included bilateral level VI LN clearance. Lymph nodes in central compartment, including all perithyroidal and paratracheal soft tissue and LNs with borders extending superiorly to the hyoid bone, inferiorly to the innominate artery, and laterally to the common carotid arteries, were dissected following total thyroidectomy. This compartment includes the pre- and paratracheal nodes, precricoid (Delphian) node, and the perithyroidal nodes, including the LNs along the recurrent laryngeal nerve. The prelaryngeal LNs, sitting directly anteriorly to the cricothyroid membrane between the cricothyroid muscles, were dissected at the time of mobilization of the thyroid pyramidal lobe and isthmus. In each patient, the recurrent laryngeal nerves (RLN) were exposed. We prefer to identify the RLN just caudal to the point where it crosses the inferior thyroid artery and to dissect it in both directions: caudally to the mediastinum and cranially to the cricothyroid junction. Intraoperative electrophysiological nerve monitoring was also employed in most of the patients. All vessels were ligated close to the thyroid gland especially the branches of the inferior thyroid artery. The parathyroid glands were dissected meticulously from the thyroid gland, and an effort was made to identify all four parathyroids and preserve as many as possible in situ. Any parathyroid gland that could not be preserved or devascularized during dissection was reimplanted into the sternocleidomastoid muscle. Bilateral thymectomy (upper poles of thymus gland) included into the dissection material when (1) central LN metastases documented preoperatively or (2) both superior and at least one of the inferior parathyroid glands ideally preserved along with their vascular integrity.

\subsection{Pathologic Analysis}

Macroscopic examinations were done by same experienced pathologist. Lymph nodes were dissected by manual palpation in the CLND specimen and rest of the fibro-adipose tissue submitted for pathological process for possible microscopic lymph nodes. All specimens were fixed in $10 \%$ formalin solution and were preserved in paraffin 
blocks, which were cut serially in sections of $3 \mu \mathrm{m}$ thickness and deparaffinized sections were stained with hematoxylin-eosin. Lymph nodes were assessed by routine histological examination and no additional techniques were used. The number of microscopic lymph nodes was assessed according to previous report by Parkash et al [11]. Organized collection of lymphocytes and lymphoid follicles with well defined capsule were counted as a lymph node. If multiple nodes were submitted in a single cassette and there were 2 lymph nodes in a single fragment of fat, we counted this as 2 nodes. If the gross description said a single node was determined and microscopic observing showed 1 grossly remarkable node and 1 minor lymph node, we counted this as 2 nodes. If gross examination revealed as 2 or more $\mathrm{cm}$ diameters aggregate of adipose tissue which completely submitted, and the slide shows multiple lymph nodes, we counted this as 1 lymph node [11].

Each patient's clinicopathologic information, including age, gender, number of total LNs and metastatic LNs, presence of TT in dissection material and existence of underlying CLT were obtained from pathology reports. CLT was diagnosed as pathological specimens regardless of preoperative anti-thyroid antibodies and ultrasonographic findings. The diagnosis of CLT was defined as the presence of extensive lymphocytic infiltrations in the thyroid parenchyma, with well-developed germinal centers and presence of oxyphilic cells metaplasia in thyroid follicular cells. Assessment of the clinical stage of PTC was based on the American Joint Committee on Cancer (AJCC) TNM system [12].

Patients divided into three different LN score according to total number of LNs in CLND material as follows: low LN score (1 - 5 lymph nodes), intermediate LN score (6 - 10 lymph nodes), and high LN score (>10 lymph nodes).

\subsection{Statistical Analyses}

SPSS 12.0 for windows (SPSS Inc., Chicago, IL) was used for statistical analysis. The wilcoxon test, were used to analyze the statistical relationship between total lymph node number in CLND material and existence of CLT and TT. A two-tailed $p$ less than 0.05 was considered statistically significant.

\section{Results}

A total of 153 patients (mean age $41 \pm 11 \mathrm{yr}$, range 15 - 78) with PTC were evaluated in this study. The clinical and pathologic characteristics of the study group are reported in Table 1. Histopathologic examination revealed CLT and TT in CLND materials in 70 and 63 patients, respectively. Distribution of patients according to presence of CLT and TT in dissection material was summarized in Table 2.

The mean number of LNs removed from central compartment was mean $11 \pm 5.7$ (2 - 49). LN numbers in CLND materials and statistical analysis results for CLT and TT groups as well as subgroups were given in Table 3. Total number of LNs in CLND materials was significantly higher in CLT $(+)$ and TT $(+)$ groups $(\mathrm{p}<0.001)$, independently. Existence of CLT associated with significantly higher number of central LNs in both TT 
Table 1. Demographic and pathologic characteristics of study group.

\begin{tabular}{|c|c|c|}
\hline Characteristics & $n$ & $\%$ \\
\hline \multicolumn{3}{|l|}{ Gender } \\
\hline Male & 29 & 19 \\
\hline Female & 124 & 81 \\
\hline \multicolumn{3}{|l|}{ Age at diagnosis (years) } \\
\hline$<45$ & 101 & 66 \\
\hline$\geq 45$ & 52 & 34 \\
\hline \multicolumn{3}{|l|}{ Histopathologc type } \\
\hline Well differentiated type & 140 & 91.5 \\
\hline Poor prognostic types ${ }^{*}$ & 13 & 8.5 \\
\hline \multicolumn{3}{|l|}{ T stage } \\
\hline T1a & 77 & 50 \\
\hline $\mathrm{T} 1 \mathrm{~b}$ & 58 & 38 \\
\hline $\mathrm{T} 2$ & 10 & 6.5 \\
\hline T3 & 8 & 5.5 \\
\hline Central LN metastasis (+/-) & $61 / 92$ & $40 / 60$ \\
\hline Lateral LN metastasis $(+/-)$ & $23 / 130$ & $15 / 85$ \\
\hline Presence of CLT (+/-) & $70 / 83$ & $46 / 54$ \\
\hline Existence of TT in CLND material (+/-) & $63 / 90$ & $41 / 59$ \\
\hline
\end{tabular}

LN: lymph node; CLND: central lymph node dissection; CLT: chronic lymphocytic thyroiditis; TT: thymic tissue, ${ }^{*}$ AJCC TNM system 2010 [12].

Table 2. The distribution of patients according to co-occurrence of chronic lymphocytic thyroiditis and presence of thymic tissue in central lymph node dissection material.

\begin{tabular}{cccc}
\hline & CLT (-) & CLT (+) & $\boldsymbol{n}$ \\
\hline TT (-) & 50 & 40 & 90 \\
TT (+) & 33 & 30 & 63 \\
$\boldsymbol{n}$ & 83 & 70 & \\
\hline
\end{tabular}

CLT: chronic lymphocytic thyroiditis; TT: thymic tissue.

Table 3. The relationship between the mean number of central lymph node and presence of chronic lymphocytic thyroiditis and thymic tissue in all groups and subgroups.

\begin{tabular}{cccccc}
\hline & CLT (-) & CLT (+) & $p$ (subgroups) & TT Group & p (TT group) \\
\hline TT (-) & $6(3-12)$ & $11.0(5.5-15)$ & 0.007 & $8(4-14)$ & $<0.001$ \\
TT (+) & $9.5(7-13.25)$ & $14(9-28.5)$ & 0.006 & $11(8-16)$ & \\
$p$ (subgroups) & 0.006 & 0.024 & & & \\
CLT Group & $7.5(4-13)$ & $12(8-16.25)$ & & & \\
p (CLT group) & $<0.001$ & & & \\
\hline
\end{tabular}

CLT: chronic lymphocytic thyroiditis; TT: thymic tissue.

(+) and TT (-) subgroups. On the other hand, LN numbers did not differ statistically between CLT $(+)$ and TT $(-)$ subgroups in existence of TT.

Both existence of CLT and TT associated with more patients with intermediate and 
high LN scores. LN scores according to total number of LNs in CLND material were given in Table 4.

LN metastases in central compartment were found in $40 \%$ of patients $(n=61)$. Of these, 30 patients had underlying CLT. Though it was not one of the main objectives of this study, metastatic LN ratio (metastatic LN/total LN) was significantly lower in CLT (+) group, comparing to CLT $(-)$ group $(16.8 \% \pm 12.2 \%$ vs $46.4 \% \pm 27.5 \%$; $\mathrm{p}<0.001)$. Demographic and pathologic characteristics of study group according to co-existence of CLT were summarized in Table 5. No thymic metastases were found in central dissection materials.

Table 4. Lymph node scores according to total number of lymph nodes in central lymph node dissection material in all groups and subgroups.

\begin{tabular}{ccccc}
\hline & Low LN score & Intermediate LN score & High LN score & $p$ \\
\hline CLT (+) & 8 & 17 & 45 & 0.016 \\
CLT (-) & 22 & 32 & 29 & \\
TT (+) & 5 & 23 & 35 & 0.017 \\
TT (-) & 26 & 26 & 38 & \\
\hline
\end{tabular}

CLT: chronic lymphocytic thyroiditis; TT: thymic tissue.

Table 5. Demographic and pathologic characteristics of study group according to co-existence of CLT.

\begin{tabular}{|c|c|c|c|}
\hline Characteristics & $\mathrm{n}(\mathrm{CLT}+)$ & n (CLT-) & $p$ value \\
\hline \multicolumn{4}{|l|}{ Gender } \\
\hline Male & 7 & 24 & \\
\hline Female & 63 & 59 & \\
\hline \multicolumn{4}{|l|}{ Age at diagnosis (years) } \\
\hline$<45$ & 46 & 52 & \\
\hline$\geq 45$ & 24 & 31 & \\
\hline \multicolumn{4}{|l|}{ Histopathologc type } \\
\hline Well differentiated types & 64 & 76 & \\
\hline Poor prognostic types ${ }^{\star}$ & 6 & 7 & \\
\hline \multicolumn{4}{|l|}{ T stage } \\
\hline T1a & 33 & 44 & \\
\hline $\mathrm{T} 1 \mathrm{~b}$ & 30 & 28 & \\
\hline $\mathrm{T} 2$ & 2 & 8 & \\
\hline $\mathrm{T} 3$ & 3 & 5 & \\
\hline \multicolumn{4}{|l|}{ N stage } \\
\hline No & 35 & 57 & \\
\hline N1a & 23 & 38 & \\
\hline $\mathrm{N} 1 \mathrm{~b}$ & 9 & 14 & \\
\hline $\begin{array}{l}\text { Number of LN in CLND material } \\
\qquad(\text { mean } \pm \text { SD })\end{array}$ & $12(8-16.25)$ & $7.5(4-13)$ & $<0.001$ \\
\hline $\begin{array}{c}\text { Metastatic LN ratio (\%) } \\
(\text { mean } \pm \mathrm{SD})\end{array}$ & $16.8 \pm 12.2$ & $46.4 \pm 27.5$ & $<0.001$ \\
\hline
\end{tabular}

LN: lymph node; CLND: central lymph node dissection; CLT: chronic lymphocytic thyroiditis; TT: thymic tissue, *AJCC TNM system 2010 [12]. 


\section{Discussion}

LN metastases are often detected at the time of initial diagnosis or during follow-up of patients with PTC. Efforts were concentrated on the ability to timely identify the presence of cervical LN metastasis prior first surgery for PTC, to justify an additional and more radical surgical procedure. Nevertheless, it remains difficult to assess central compartment nodal status preoperatively with high precision [13] [14]. Despite normal preoperative physical examination and ultrasonography, the incidence of LN metastasis in central compartment is high (29.4\%) even in microcarcinomas [4]. Rates similar to this and higher have been described by some others [15] [16]. However, routine prophylactic CLND with total thyroidectomy in clinically node negative patients still subject of an ongoing debate mostly due to increased rates of transient and permanent hypoparathyroidism. Though re-operations associate with a higher complication rates comparing to initial surgery, revision surgery still represents the main treatment for known recurrent/persistent disease in central compartment [17]. Being aware of higher complication rates of re-operations [18], it has been advocated that efforts should be made to avoid central compartment reoperation by performing a definitive and more radical initial surgical treatment. Beside providing objective information about LN status, CLND has also potential to reduce postoperative Tg levels and local recurrence rates [4].

The LN involvement in central compartment is one of the main determinants of accurate staging in PTC patients [19]. The $\mathrm{N}$ has traditionally been based on the presence or absence of positive LNs, unrelated to the number of positive LNs and to the total number of LNs detected in existing staging systems [12]. Recent studies have introduced, however, that the number and size of central LN metastases as well as extranodal involvement may also be correlated with both lateral LN metastases and central neck recurrences [7] [20]-[22]. Clinically no apparent detectable metastatic lymph nodes, so called cN0, for PTC actually declares Nx status according to TNM classification system of the AJCC. Therefore, correct staging of central compartment in the absence of gross nodal involvement requires pathologic assessment.

To accurately determine the nodal status in central compartment, the extent of central lymph node dissection and macroscopic examination of dissection materials should be adequate. The number of central compartment lymph nodes should also be known to decide on adequacy of central dissection materials. Although the adequate number of lymph nodes yet to be defined for CLND, the number of central compartment LNs and adequacy criteria have been previously described in several studies [20] [21] [23]. Tavares et al studied the anatomic distribution of the lymph nodes in the central compartment of the neck by dissection of 30 fresh cadavers. They reported that the number of central lymph nodes varied from 2 to 42 [24]. According to the protocol of the College of American Pathologist, histological examination of a selective neck dissection specimen includes 6 or more lymph nodes. However, this number represents total LNs in dissection material and adequacy criteria for central LN number are not specified separately [25]. 
The variability in the number of LNs in central compartment might be explained by several factors including experience of surgeon, surgical technique used, pathologic process and interobserver variability among pathologist as well as nonneoplastic thyroid pathologies. The total number of nodes found is important since it reflects both the completeness of the node dissection or sampling and the care with which the pathologic examination was carried out.

The number of LNs in central compartment varied from 2 to 49 (mean $11 \pm 5.7$ ) with standardized surgical and pathologic approach in our study group.

With regard to the number of LNs, there were significant differences $(\mathrm{p}<0.001)$ between CLT (+) and CLT ( - ) groups. Mean numbers of central LNs in CLT (+) and CLT (-) groups were 12 (8 - 16.25) and 7.5 (4 - 13), respectively. Previous reports also introduced that more central LNs were removed in PTC with CLT mainly due to increased number of benign hyperplastic cervical LNs [26]-[29]. Kim et al. have also reported a negative association between presence of CLT and central LN metastasis suggesting that coexistence of PTC with CLT might point out less aggressive features [27] [28]. In our study group, the mean number of central metastatic LNs $(p=0.306)$ and metastatic LNs ratio was also lower in CLT $(+)$ group comparing to CLT $(-)$ one (p < 0.001). It is not clear that lower metastatic rates in CLT (+) group is simply due to increased number of benign hyperplastic LNs in central compartment and hence sampling error or truly protective effect of CLT. But, data from our study group as well as previous reports shows that existence of CLT consistently associate higher central LNs in dissection material. Therefore, it may be possible to conclude that upper limit of adequacy for LN numbers would be also higher $(>10)$ in PTC patients with CLT for optimal definition of central compartment LN status.

The other determinant for adequacy of CLND that has been analyzed in our study was the presence of TT. Since thymus constitutes the inferior boundaries of central compartment, thymic tissue frequently seen in central dissection materials. TT was seen in central dissection materials of 63 patients in our series. The LN numbers in central compartment were significantly higher in TT $(+)$ group $(\mathrm{p}<0.001)$ indicating more effective dissection. Mean numbers of central LNs in TT $(+)$ and TT $(-)$ groups were 8 (4 - 14) and $11(8$ - 16), respectively. This result indicated that the presence of TT in CLND materials might reflect the extent and adequacy of CLND and, consequently leads to correct pathologic staging. Highest mean LN numbers reached in study group was 14 (9 - 28.5) and found in CLT (+) and TT (+) subgroup, as expected.

\section{Conclusion}

In conclusion, co-existence of PTC with CLT is quite common in routine clinical practice and results in higher LN number in central dissection material if performed, mainly due to benign hyperplastic LNs. This feature should be taken into consideration during the pathologic examination of the central dissection materials for PTC, as well as interpretation of results. The $\mathrm{LN}$ numbers in central compartment materials that would have been ideal otherwise might be suboptimal in the presence of CLT. Therefore, 
adequacy criteria in assessing central compartment nodal status would be different in patients with CLT. We recommend that the cut-off number for adequacy of central compartment LN numbers would be at least 10 , which is almost double than value suggested by the existing staging systems, if specimens have CLT. The other main result of this paper is that the existence of thymus in central compartment dissection material might be an important factor suggesting sufficient $\mathrm{LN}$ resection.

\section{Acknowledgements}

The authors thank to Dr. Muhittin A. Serdar who helped with the statistically analysis.

\section{Author Disclosure Statement}

The authors declare that there is no conflict of interest.

\section{References}

[1] Fritze, D. and Doherty, G.M. (2010) Surgical Management of Cervical LNs in Differentiated Thyroid Cancer. Otolaryngologic Clinics of North America, 43, 285-300. http://dx.doi.org/10.1016/j.otc.2010.01.005

[2] Sywak, M., Cornford, L., Roach, P., Stalberg, P., Sidhu, S. and Delbridge, L. (2006) Routine İpsilateral Level VI Lymphadenectomy Reduces Postoperative Thyroglobulin Levels in Papillary Thyroid Cancer. Surgery, 140, 1000-1005; Discussion, 1005-1007. http://dx.doi.org/10.1016/j.surg.2006.08.001

[3] Lundgren, C.I., Hall, P., Dickman, P.W. and Zedenius, J. (2006) Clinically Significant Prognostic Factors for Differentiated Thyroid Carcinoma: A Population-Based, Nested CaseControl Study. Cancer, 106, 524-531. http://dx.doi.org/10.1002/cncr.21653

[4] Lee, J., Song, Y., Soh, E.Y. (2014) Central lymph Node Metastasis İs an İmportant Prognostic factor in Patients with Papillary Thyroid Microcarcinoma. Journal of Korean Medical Science, 29, 48-52. http://dx.doi.org/10.3346/jkms.2014.29.1.48

[5] Stulak, J.M., Grant, C.S., Farley, D.R., et al. (2006) Value of Preoperative Ultrasonography in the Surgical Management of İnitial and Reoperative Papillary Thyroid Cancer. Archives of Surgery, 141, 489-494. http://dx.doi.org/10.1001/archsurg.141.5.489

[6] LiVolsi, V.A. and Baloch, Z.W. (2012) On Pathology Reports of Thyroid Cancer Specimens: What Should the Clinician Expect? Thyroid, 22, 563-565. http://dx.doi.org/10.1089/thy.2012.2206.ed

[7] Scherl, S., Mehra, S., Clain, J., et al. (2014) The Effect of Surgeon Experience on the Detection of Metastatic Lymph Nodes in the Central Compartment and the Pathologic Features of Clinically Unapparent Metastatic Lymph Nodes: What Are We Missing When We Don't Perform Prophylactic Dissection Of Central Compartment Lymph Nodes in Papillary Thyroid Cancer. Thyroid, 24, 1282-1288. http://dx.doi.org/10.1089/thy.2013.0600

[8] Pai, S.I. and Tufano, R.P. (2008) Central Compartment Neck Dissection for Thyroid Cancer. Technical considerations. ORL Journal for Otorhinolaryngology and Its Related Specialties, 70, 292-297. http://dx.doi.org/10.1159/000149831

[9] Lang, B.H., Yih, P.C., Shek, T.W., Wan, K.Y., Wong, K.P. and Lo, C.Y. (2012) Factors Affecting the Adequacy of Lymph Node Yield in Prophylactic Unilateral Central Neck Dissection for Papillary Thyroid Carcinoma. Journal of Surgical Oncology, 106, 966-971. http://dx.doi.org/10.1002/jso.23201 
[10] Son, Y.I., Jeong, H.S., Baek, C.H., et al. (2008) Extent of Prophylactic Lymph Node Dissection in the Central Neck Area of the Patients with Papillary Thyroid Carcinoma: Comparison of Limited versus Comprehensive Lymph Node Dissection in a 2-Year Safety Study. Annals of Surgical Oncology, 15, 2020-2026. http://dx.doi.org/10.1245/s10434-008-9928-8

[11] Parkash, V., Bifulco, C., Feinn, R., Concato, J. and Jain, D. (2010) To Count and How to Count, That İs the Question İnterobserver and İntraobserver Variability among Pathologists in Lymph Node Counting. American Journal of Clinical Pathology, 134, 42-49. http://dx.doi.org/10.1309/AJCPO92DZMUCGEUF

[12] AJCC Cancer Staging Manual. Springer, New York, 2010.

[13] Choi, J.S., Kim, J., Kwak, J.Y., Kim, M.J., Chang, H.S. and Kim, E.K. (2010) Preoperative Staging of Papillary Thyroid Carcinoma: Comparison of Ultrasound İmaging and CT. American Journal of Roentgenology, 193, 871-878. http://dx.doi.org/10.2214/AJR.09.2386

[14] Shimamoto, K., Satake, H., Sawaki, A., Ishigaki, T., Funahashi, H. and Imai, T. (1998) Preoperative Staging of Thyroid Papillary Carcinoma with Ultrasonography. European Journal of Radiology, 29, 4-10. http://dx.doi.org/10.1016/S0720-048X(97)00184-8

[15] Bonnet, S., Hartl, D., Leboulleux, S., et al. (2009) Prophylactic Lymph Node Dissection for Papillary Thyroid Cancer Less than $2 \mathrm{~cm}$ : İmplications for Radioiodine Treatment. The Journal of Clinical Endocrinology \& Metabolism, 94, 1162-1167. http://dx.doi.org/10.1210/jc.2008-1931

[16] Alzahrani, A.S., Raef, H., Sultan, A., et al. (2002) Impact of Cervical Lymph Node Dissection on Serum TG and the Course of Disease in TG-Positive, Radioactive Iodine Whole Body Scan-Negative Recurrent/Persistent Papillary Thyroid Cancer. Journal of Endocrinological Investigation, 25, 526-531. http://dx.doi.org/10.1007/BF03345495

[17] Jeon, M.J., Yoon, J.H., Han, J.M., et al. (2013) The Prognostic Value of the Metastatic Lymph Node Ratio and Maximal Metastatic Tumor Size in Pathological N1a Papillary Thyroid Carcinoma. European Journal of Endocrinology, 168, 219-225. http://dx.doi.org/10.1530/EJE-12-0744

[18] Kim, M.K., Mandel, S.H., Baloch, Z., et al. (2004) Morbidity Following Central Compartment Reoperation for Recurrent or Persistent Thyroid Cancer. Archives of Otolaryngology_Head and Neck Surgery, 130, 1214-1216. http://dx.doi.org/10.1001/archotol.130.10.1214

[19] Cooper, D.S., Doherty, G.M., Haugen, B.R., et al. (2009) Revised American Thyroid Association ManagementGuidelines for Patients with Thyroid Nodulesand Differentiated Thyroid Cancer. The American Thyroid Association (ATA) Guidelines Taskforce on Thyroid Nodules and Differentiated Thyroid Cancer. Thyroid, 19, 1167-1214. http://dx.doi.org/10.1089/thy.2009.0110

[20] Fu, J.Y., Wu, Y., Wang, Z.Y., An, Y., Sun, T.Q. and Xiang, J. (2007) Clinical and Pathological Analysis of Central Compartment Dissection in Patients with Papillary Thyroid Cancer with Negative Clinical Lymph Node Metastasis. Chinese Journal of Surgery, 45, 470-472.

[21] Pereira, J.A., Jimeno, J., Miquel, J., et al. (2005) Nodal Yield, Morbidity, and Recurrence after Central Neck Dissection for Papillary Thyroid Carcinoma. Surgery, 138, 1095-1100. http://dx.doi.org/10.1016/j.surg.2005.09.013

[22] Zeng, R.C., Zhang, W., Gao, E.L., Cheng, P., Huang, G.L., Zhang, X.H. and Li, Q.H. (2014) Number of Central Lymph Node Metastasis for Predicting Lateral Lymph Node Metastasis in Papillary Thyroid Microcarcinoma. Head \& Neck, 36, 101-106.

http://dx.doi.org/10.1002/hed.23270 
[23] Hartl, D.M., Leboulleux, S., Al Ghuzlan, A., et al. (2012) Optimization of Staging of the Neck with Prophylactic Central and Lateral Neck Dissection for Papillary Thyroid Carcinoma. Annals of Surgery, 255, 777-783. http://dx.doi.org/10.1097/SLA.0b013e31824b7b68

[24] Tavares, M.R., Shiomi da Cruz, J.A., Waisberg, D.R., et al. (2014) Lymph Node Distribution in the Central Compartment of the Neck: An Anatomic Study. Head \& Neck, 36, 1425-1430. http://dx.doi.org/10.1002/hed.23469

[25] College of American Pathologists (2014) College of American Pathologists Cancer Protocols and Checklists. http://www.cap.org/

[26] Brancato, D., Citarrella, R., Richiusa, P., Amato, M.C., Vetro, C. and Galluzzo, C.G. (2013) Neck Lymph Nodes in Chronic Autoimmune Thyroiditis: The Sonographic Pattern. Thyroid, 23, 173-177. http://dx.doi.org/10.1089/thy.2012.0375

[27] Kim, Y.S., Choi, H.J. and Kim, E.S.J. (2013) Papillary Thyroid Carcinoma with Thyroiditis: Lymph Node Metastasis, Complications. Journal of the Korean Surgical Society, 85, 20-24. http://dx.doi.org/10.4174/jkss.2013.85.1.20

[28] Kim, S.S., Lee, B.J., Lee, J.C., et al. (2011) Coexistence of Hashimoto's Thyroiditis with Papillary Thyroid Carcinoma: The Influence of Lymph Node Metastasis. Head \& Neck, 33, 1272-1277. http://dx.doi.org/10.1002/hed.21594

[29] Sahlmann, C.O., Meller, J., Siggelkow, H., et al. (2012) Patients with Autoimmune Thyroiditis. Prevalence of Benign Lymphadenopathy. Nuklearmedizin, 51, 223-227.

http://dx.doi.org/10.3413/Nukmed-0484-12-03

\section{Submit or recommend next manuscript to SCIRP and we will provide best service for you:}

Accepting pre-submission inquiries through Email, Facebook, LinkedIn, Twitter, etc.

A wide selection of journals (inclusive of 9 subjects, more than 200 journals)

Providing 24-hour high-quality service

User-friendly online submission system

Fair and swift peer-review system

Efficient typesetting and proofreading procedure

Display of the result of downloads and visits, as well as the number of cited articles

Maximum dissemination of your research work

Submit your manuscript at: http://papersubmission.scirp.org/ 